\title{
EL FORO DE SALUD MENTAL Y \\ LA SALUD COMUNITARIA
}

\begin{abstract}
“¿Qué es la salud comunitaria?
“... todo intento de definición de salud comunitaria nos lleva a repensar sobre los inicios y constitución de es a comunidad, en la cual la salud aparecería como un producto elaborado por ella misma en diversos momentos de su historia”.
\end{abstract}

Armando BAULEO*

La colaboración con otras organizaciones con las que hemos compartido objetivos y valores de nuestra Asociación ha estado siempre presente en nuestras actividades. Este hecho lo hemos visto plasmados en numerosas ocasiones: mesas redondas, ponencias en congresos, grupos de trabajo, por citar sólo algunos.

En este contexto ha tenido lugar hace unas semanas el primer encuentro entre varias organizaciones para formalizar el Foro de Salud Mental. Han aceptado la invitación a esta convocatoria de nuestra Asociación varias organizaciones de profesionales sanitarios, de usuarios -familiares y pacientes- y algún colectivo ciudadano.

El Foro tiene como objetivo poner en común, reflexionar y expresar nuestra preocupación sobre la situación actual de la Salud Mental en el ámbito del Estado. Los recortes que han ido apareciendo en algunas Comunidades Autónomas y las previsiones de futuro próximas nos hacen temer que lo avanzado en las últimas décadas en materia de derechos sanitarios y sociosanitarios, puede verse en riesgo y devolvernos a situaciones pasadas.

Nos preocupa en especial el cuestionamiento del estado del bienestar y sus derechos, y el riesgo de perder un modelo de atención a la salud, basado en una asistencia pública, universal, gratuita, participativa e integradora.

La salud mental se ha incorporado e integrado en los servicios sanitarios del país hace relativamente escaso tiempo, saliendo de su marginación secular y con una consistencia frágil aún. Por esta razón, la situación general existente pone en peligro lo conseguido en un ámbito muy vulnerable, tanto por la población que atiende, como por inestabilidad de su estructura.

La merma de recursos o la transferencia de la gestión de servicios a manos privadas rompen con el proceso iniciado hace años en la mayoría de las Comuni-

\footnotetext{
Armando Bauleo. Notas de psicología y psiquiatría social. Ed. Atuel S.A. Buenos Aires, 1988.
} 
dades Autónomas. Aunque a distintas velocidades y con diferencias en la organización, en ellas se iba instaurando un modelo de atención a la salud mental con las características de la llamada salud mental comunitaria, siguiendo las directrices de la OMS y lo que la evidencia científica y la eficiencia demostrada van implantando en el mundo.

Pero estamos asistiendo no sólo a una merma de recursos o cambios en los modelos de gestión. Estamos ante un cambio de modelo de salud (mental). O lo que es lo mismo, a un cambio de modelo social.

Hablar de salud mental comunitaria, o simplemente salud comunitaria, implica: una organización de los servicios teniendo en cuenta las características epidemiológicas y sociológicas de un territorio, de manera que garantice la universalidad, la equidad y la accesibilidad de las prestaciones, desde la prevención, promoción de la salud hasta la rehabilitación; el objetivo de implementar prestaciones de calidad a través de la gestión clínica, de manera que la auténtica eficiencia es la eficacia, o sus eslabones intermedios, la efectividad; y el trabajo en red, como resultado de la confluencia de profesionales de diferentes ámbitos, sanitarios y no sanitarios, institucionales o no, pero también de usuarios, pacientes y familiares.

Territorio, cultura y personas están pues en la construcción de las respuestas a los problemas de salud, comenzando por la propia definición de salud. Son palabras claves de esta forma de entender el ámbito de la salud comunitaria, participación, empoderamiento, rendición de cuentas, conocimientos, experiencias, de todos y cada uno de los protagonistas. Y como soporte de todo este quehacer se requiere un sujeto en, desde, y para las relaciones. Un sujeto con historia, también valores y derechos.

Los recortes, la privatización, representan aspectos de otro tipo de modelo, basado sólo en servicios para la enfermedad, decidida de antemano en la oferta asistencial y sin tener en cuenta las necesidades de la población, por tanto insolidario; en función del beneficio de quien gestiona y donde la competencia sustituye a la cooperación y el mercado a las personas. El sujeto con historia, valores y derechos es sustituido por el hombre mercancía.

Por estas razones, se hace más necesario el Foro de Salud (Mental). Es otra forma de escenificar lo que desde el ámbito de nuestra Asociación llevamos proclamando y practicando hace ya mucho tiempo: lo multiprofesional, lo interdisciplinario, la cooperación, la participación y, siempre, el compromiso. De esta manera formalizamos alianzas con todos aquellos con los que construimos en el quehacer de todos los días la salud comunitaria. 